\title{
ON THE SIZE OF LATTICE SIMPLICES WITH A SINGLE INTERIOR LATTICE POINT
}

\author{
Gennadiy Averkov*
}

November 21, 2018

\begin{abstract}
Let $\mathcal{T}^{d}$ be the set of all $d$-dimensional simplices $T$ in $\mathbb{R}^{d}$ with integer vertices and a single integer point in the interior of $T$. It follows from a result of Hensley that $\mathcal{T}^{d}$ is finite up to affine transformations that preserve $\mathbb{Z}^{d}$. It is known that, when $d$ grows, the maximum volume of the simplices $T \in \mathcal{T}^{d}$ becomes extremely large. We improve and refine bounds on the size of $T \in \mathcal{T}^{d}$ (where by the size we mean the volume or the number of lattice points). It is shown that each $T \in \mathcal{T}^{d}$ can be decomposed into an ascending chain of faces $G_{1} \subseteq \cdots \subseteq G_{d}=T$ such that, for every $i \in\{1, \ldots, d\}, G_{i}$ is $i$-dimensional and the size of $G_{i}$ is bounded from above in terms of $i$ and $d$. The bound on the size of $G_{i}$ is double exponential in $i$. The presented upper bounds are asymptotically tight on the log-log scale.
\end{abstract}

2010 Mathematics Subject Classification: Primary 52B20; Secondary 14J45, 90C11

Keywords: barycentric coordinates; inequality; lattice-point enumerator; lattice polytope; Minkowski's fundamental theorem; simplex; volume

\section{Introduction}

For standard background from the geometry of numbers and convex geometry we refer to GL87, Sch93, Bar02, Gru07]. The cardinality of a set $X$ is denoted by $|X|$. Throughout the paper we fix a $d$-dimensional vector space $\mathbb{V}$ over $\mathbb{R}$ with $d \in \mathbb{N}$ and a lattice $\mathbb{L}$ of rank $d$ in $\mathbb{V}$. In most of the cases it will be convenient to use coordinate-free notation. However, when we use analytic expressions with matrices or the notion of volume, we fix coordinates. In such cases $\mathbb{V}$ is identified with $\mathbb{R}^{d}$, the set of columns of $d$ real numbers. If $\mathbb{M}$ is a lattice of rank $i \in\{1, \ldots, d\}$, then the determinant of $\mathbb{M}$ (denoted by $\operatorname{det} \mathbb{M}$ ) is the $i$-dimensional volume of any Dirichlet cell of $\mathbb{M}$. A subset $P$ of $\mathbb{V}$ is said to be a lattice polytope (with respect to the lattice $\mathbb{L}$ ) if $P$ is the convex hull of finitely many points of $\mathbb{L}$. Let $P$ be an $i$-dimensional lattice polytope in $\mathbb{V}$, where $i \in\{1, \ldots, d\}$. Then by $\operatorname{vol}(P)$ we denote the $i$-dimensional volume of $P$. We also use the $i$-dimensional normalized volume of $P$ defined by

$$
\operatorname{vol}_{\mathbb{L}}(P):=\frac{\operatorname{vol}(P)}{\operatorname{det} \mathbb{M}}
$$

\footnotetext{
*Institute for Mathematical Optimization, Faculty of Mathematics, University of Magdeburg, Universitätsplatz 2, 39106 Magdeburg, Germany. Email: averkov@math.uni-magdeburg.de
} 
where $\mathbb{M}:=\mathbb{L} \cap X$ and $X$ is the linear hull of vectors $x-y$ with $x, y \in P$. We remark that $\operatorname{vol}_{\mathbb{L}}(P)$ does not depend on the choice of coordinates in $\mathbb{V}$.

Let $\mathcal{T}^{d}$ denote the family of all $d$-dimensional lattice simplices in $\mathbb{V}$ with precisely one interior lattice point. It was shown by Hensley [Hen83] that the volume of the elements of $\mathcal{T}^{d}$ is bounded by a constant depending only on $d$. Continuing the research initiated in [Hen83], we present bounds on the size of lattice simplices with precisely one interior lattice point.

Note that the family $\mathcal{T}^{d}$ appears naturally in algebraic geometry; see BB92, Bor00, Kas09]. Furthermore, as seen from [AWW11], $\mathcal{T}^{d}$ can be used in the study of the class $\mathcal{L}^{d}$ of inclusion-maximal $d$-dimensional lattice polytopes without interior lattice points. The elements of $\mathcal{L}^{d}$ are important for the cutting-plane theory in integer and mixed integer optimization (see [AWW11, DPW10] and the references therein).

We prove the following theorem, which is our main tool.

Theorem 1.1. Let $T \in \mathcal{T}^{d}$. Let $\beta_{i}$ with $i \in\{0, \ldots, d\}$ be the barycentric coordinates of the unique interior lattice point of $T$. Then, for every partition $(I, J)$ of $\{0, \ldots, d\}$, one has

$$
\sum_{i \in I} \beta_{i} \geq \prod_{j \in J} \beta_{j}
$$

In Theorem 1.1 without loss of generality one can assume $\beta_{0} \geq \cdots \geq \beta_{d}>0$. Then, using (1.1), it is possible to determine lower bounds for $\beta_{i}$ 's in terms of $i$ and $d$. As a consequence we obtain the following result on the maximum size of simplices from $\mathcal{T}^{d}$.

Theorem 1.2. Let $d \in \mathbb{N}$. Then the following statements hold.

I. For every $T \in \mathcal{T}^{d}$ there exist faces $G_{1} \subseteq \cdots \subseteq G_{d}=T$ of $T$ such that, for every $i \in\{1, \ldots, d\}, G_{i}$ is $i$-dimensional and satisfies

$$
\begin{aligned}
\operatorname{vol}_{\mathbb{L}}\left(G_{i}\right) & \leq \frac{1}{i !}(d+1)^{2^{i}-1}, \\
\left|G_{i} \cap \mathbb{L}\right| & \leq i+(d+1)^{2^{i}-1} .
\end{aligned}
$$

II. There exists $T^{\prime} \in \mathcal{T}^{d}$ and faces $G_{1} \subseteq \cdots \subseteq G_{d}=T^{\prime}$ of $T^{\prime}$ such that, for every $i \in\{1, \ldots, d\}, G_{i}$ is $i$-dimensional and satisfies

$$
\begin{aligned}
\operatorname{vol}_{\mathbb{L}}\left(G_{i}\right) & \geq \frac{1}{i !}\left(2^{2^{i-1}}-1\right), \\
\left|G_{i} \cap \mathbb{L}\right| & \geq 2^{2^{i-2}} .
\end{aligned}
$$

Loosely speaking, Theorem 1.2 asserts that the largest simplices from $\mathcal{T}^{d}$ are extremely anisotropic. Part I contains the main message of Theorem 1.2. Part II is given as a complement estimating the quality of the upper bounds from Part I. For $d \in \mathbb{N}$ let

$$
v(d):=\sup _{T \in \mathcal{T}^{d}} \operatorname{vol}_{\mathbb{L}}(T)
$$

By Theorem 1.2, we obtain $v(d)=(d+1)^{O\left(2^{d}\right)}$. This improves the best available bound $v(d)=\left(2^{d}\right)^{O\left(2^{d}\right)}$, which can be found in [Pik01, (10)]. 
Let us give a short overview of results related to Theorem 1.2. The study of upper bounds on the size of lattice polytopes with a fixed positive number of interior lattice points was initiated in [Hen83] and continued in [LZ91] and [Pik01. The sources [Law91] and [BB92] present qualitative arguments which show $v(d)<+\infty$ for every $d \in \mathbb{N}$ (see also [Bor00, §4] and [Pik01, §7]). Analoga of the results from [Hen83, LZ91, Pik01] for lattice polytopes without interior lattice points were given in [HNP09, AWW11, NZ11]. The sources [Rab89] and Kas10] provide classification of $d$-dimensional lattice polytopes with precisely one interior lattice point for the cases $d=2$ and $d=3$, respectively. Inequalities involving the area of lattice polygons can be found in [Ehr55, Sco76, HS09].

\section{Preliminaries}

The abbreviations aff, conv and int stand for the affine hull, convex hull and interior, respectively. The origin of $\mathbb{V}$ is denoted by $o$. By $e_{1}, \ldots, e_{d}$ we denote the standard basis of $\mathbb{R}^{d}$. The $l_{\infty}$-norm is denoted by $\|\cdot\|_{\infty}$. We shall use the following three well-known results from the geometry of numbers.

Theorem 2.1. (Minkowski's fundamental theorem.) Let $U$ be an open convex set in $\mathbb{V}$ with $U=-U$ and $\operatorname{vol}_{\mathbb{L}}(U)>2^{d}$. Then $U$ contains a nonzero element of $\mathbb{L}$.

Theorem 2.2. Let $A$ be a real $d \times d$ matrix with $0<|\operatorname{det} A|<1$. Then there exists $x \in \mathbb{Z}^{d} \backslash\{o\}$ satisfying $\|A x\|_{\infty}<1$.

Theorem 2.3. (Blichfeldt's theorem.) Let $K$ be a compact convex set in $\mathbb{V}$ containing

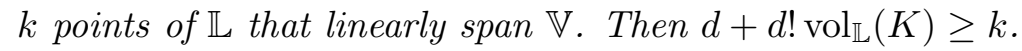

Note that Theorem 2.2 is a straightforward consequence of Theorem 2.1.

Let $T$ be a $d$-dimensional simplex in $\mathbb{V}$ and let $p_{0}, \ldots, p_{d}$ be the vertices of $T$. Then every point $x \in \mathbb{V}$ can be uniquely given by the values $\alpha_{0}, \ldots, \alpha_{d} \in \mathbb{R}$ satisfying

$$
\begin{aligned}
x & =\alpha_{0} p_{0}+\cdots+\alpha_{d} p_{d}, \\
1 & =\alpha_{0}+\cdots+\alpha_{d} .
\end{aligned}
$$

The values $\alpha_{0}, \ldots, \alpha_{d}$ are said to be the barycentric coordinates of $x$ with respect to the simplex $T$. The barycentric coordinates have the following geometric interpretation. Let $H_{i}$ be the affine hull of the facet of $T$ opposite to $p_{i}$. Then $\alpha_{i}$ is the signed weighted distance from $x$ to $H_{i}:\left|\alpha_{i}\right|$ is the ratio of the distance of $x$ to $H_{i}$ to the distance of $p_{i}$ to $H_{i}$, and $\alpha_{i}>0$ if and only if $x$ and $p_{i}$ are in the same open halfspace defined by $H_{i}$. In particular, we have:

- $x$ lies in the interior of $T$ if and only if $\alpha_{i}>0$ for all $i \in\{0, \ldots, d\}$.

- For $i \in\{0, \ldots, d\}, x \in H_{i}$ if and only if $\alpha_{i}=0$.

The barycentric coordinates $\alpha_{0}, \ldots, \alpha_{d}$ of $x$ can be found from

$$
\left[\begin{array}{ccc}
p_{0} & \cdots & p_{d} \\
1 & \cdots & 1
\end{array}\right]\left[\begin{array}{c}
\alpha_{0} \\
\vdots \\
\alpha_{d}
\end{array}\right]=\left[\begin{array}{c}
x \\
1
\end{array}\right]
$$


(We recall that in matrix expressions we assume $\mathbb{V}=\mathbb{R}^{d}$.) Since $p_{0}, \ldots, p_{d}$, are affinely independent, the $(d+1) \times(d+1)$-matrix in (2.2) is invertible. Thus, we can express $\alpha_{i}$ 's by

$$
\alpha_{i}:=e_{i}^{\top}\left[\begin{array}{ccc}
p_{0} & \cdots & p_{d} \\
1 & \cdots & 1
\end{array}\right]^{-1}\left[\begin{array}{l}
x \\
1
\end{array}\right] .
$$

It follows that for every $i \in\{0, \ldots, d\}$ we have $\alpha_{i}:=l_{i}(x)$, where $l_{i}(x)$ is an affine-linear function in $x$. (A real-valued function $f$ on $\mathbb{V}$ is called affine-linear if $f((1-\lambda) x+\lambda y)=$ $(1-\lambda) f(x)+\lambda f(y)$ for all $x, y \in \mathbb{V}, \lambda \in \mathbb{R}$.) The functions $l_{0}, \ldots, l_{d}$ are uniquely determined by

$$
l_{i}\left(p_{j}\right)=\delta_{i, j} \quad \forall i, j \in\{0, \ldots, d\},
$$

where $\delta_{i, j}$ denotes the Kronecker delta. This follows by applying $l_{i}$ to (2.1) and then using the affine-linearity.

We shall use pairs $(I, J)$ of disjoint subsets of $\{0, \ldots, d\}$ with $I \cup J=\{0, \ldots, d\}$. Such a pair $(I, J)$ is called a partition if both $I$ and $J$ are nonempty. Every face of $T$ can be represented by

$$
\begin{aligned}
F_{I} & :=\left\{x \in T: l_{i}(x)=0 \quad \forall i \in I\right\} \\
& =\operatorname{conv}\left\{p_{j}: j \in J\right\}
\end{aligned}
$$

for an appropriate pair $(I, J)$ as above. Clearly, partitions $(I, J)$ correspond to proper faces of $T$.

\section{The proofs}

\subsection{Polynomial inequalities for $\beta_{i}$ 's (proof of Theorem 1.1)}

Let $T \in \mathcal{T}^{d}$ and let $\beta_{0}, \ldots, \beta_{d}$ be the barycentric coordinates of the interior lattice point of $T$. Our aim is to provide positive lower bounds for $\beta_{i}$ 's in terms of $d$. Let us sketch how such bounds can be obtained (the idea is due to Hensley [Hen83, the proof of Lemma 2.3]). Let $p$ be the interior lattice point of $T$. One can show by contradiction that $p$ cannot be too close to the boundary of $T$ (that is, the minimal $\beta_{i}$ cannot be too small). If $p$ is close to a face $F$ of $T$, then, with the help of Minkowski's first fundamental theorem, one can determine a point $r$ in aff $F$ whose barycentric coordinates are rational numbers of the form $\frac{m_{0}}{m}, \ldots, \frac{m_{d}}{m}$ with $m_{0}, \ldots, m_{d} \in \mathbb{Z}$ and $m \in \mathbb{N}$ and such that $r$ is close to $p$. It then turns out that $q:=(m+1) p-m r$ is another integral point in the interior of $T$, yielding a contradiction to $T \in \mathcal{T}^{d}$. See also Fig. 1 for an illustration.

Proof of Theorem 1.1. Let $p$ be the interior lattice point of $T$. The point $p$ can be given by $p=\sum_{i=0}^{d} \beta_{i} p_{i}$, where $p_{0}, \ldots, p_{d}$ are appropriately indexed vertices of $T$. Without loss of generality let $I=\{t, \ldots, d\}$ with $1 \leq t \leq d$. Consider real unknowns $m_{0}, \ldots, m_{t-1}$ and $m$ and the vector

$$
\bar{m}:=\left(m_{0}, \ldots, m_{t-1}, m\right)^{\top}
$$

If

$$
\bar{m} \in \mathbb{Z}^{t+1}, m>0 \text { and } m=\sum_{i=0}^{t-1} m_{i}
$$




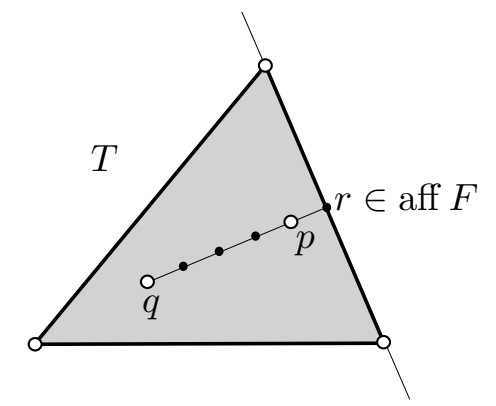

Figure 1: Using a lattice point $p \in \operatorname{int} T$ for construction of another lattice point $q \in \operatorname{int} T$ in the case that $p$ is close to a face $F$ of $T$. The argument uses a rational point $r \in$ aff $F$ which approximates $p$ sufficiently well. The point $q$ is obtained by adding $m(p-r)$ to $p$, where $m$ is a common denominator of the barycentric coordinates of $r$ (in the figure one has $m=4$ )

then

$$
r=\sum_{i=0}^{t-1} \frac{m_{i}}{m} p_{i}
$$

is an affine combination (with rational coefficients) of $p_{0}, \ldots, p_{t-1}$ and

$$
\begin{aligned}
q & :=(m+1) p-m r \\
& =(m+1) p-\sum_{i=0}^{t-1} m_{i} p_{i}
\end{aligned}
$$

is an affine combination of $p$ and $r$ belonging to $\mathbb{L}$. Below we determine a sufficient condition for the existence of values $m_{0}, \ldots, m_{t-1}, m$ as above for which the point $q$ does not coincide with $p$ and lies in the interior of $T$. If (3.1) holds, then $(m+1) \beta_{i}-m_{i}$ with $i \in\{0, \ldots, t-1\}$ and $(m+1) \beta_{i}$ with $i \in\{t, \ldots, d\}$ are the barycentric coordinates of $q$. Thus (taking into account the geometric meaning of the barycentric coordinates) we obtain that, assuming (3.1), the following conditions are equivalent:

(i) $q \in \operatorname{int} T$;

(ii) $(m+1) \beta_{i}-m_{i}>0$ for every $i \in\{0, \ldots, t-1\}$.

Using (i) $\Leftrightarrow$ (ii) we can formulate the following system of conditions on $\bar{m}$ sufficient for $q$ to be a lattice point in int $T$ with $q \neq p$ :

$$
\left\{\begin{aligned}
\bar{m} & \in \mathbb{Z}^{t+1}, \\
m & >0, \\
\frac{1}{\beta_{i}} m_{i}-m & <1 \\
m-\sum_{i=0}^{t-1} m_{i} & =0 .
\end{aligned} \quad \forall i \in\{0, \ldots, t-1\}\right.
$$


We also consider another system somewhat similar to (3.3)-(3.6):

$$
\left\{\begin{array}{rl}
\bar{m} & \in \mathbb{Z}^{t+1}, \\
\bar{m} & \neq o, \\
\left|\frac{1}{\beta_{i}} m_{i}-m\right| & <1 \\
\left|m-\sum_{i=0}^{t-1} m_{i}\right| & <1 .
\end{array} \quad \forall i \in\{0, \ldots, t-1\},\right.
$$

For (3.7)-(3.10) Theorem 2.2 can be applied, but first we investigate the relation of (3.7) - (3.10) to (3.3)-(3.6). We claim that if (3.7)-(3.10) is solvable with respect to $\bar{m}$, then also (3.3)-(3.6) is solvable with respect to $\bar{m}$. Assume that conditions (3.7)-(3.10) are fulfilled. Then $m$ is not zero. In fact, if $m=0$, then (3.9) together with (3.7) and $0<\beta_{i}<1$ implies $m_{i}=0$ for every $i \in\{0, \ldots, t-1\}$. This yields a contradiction to (3.8). Hence $m \neq 0$. The system (3.7) $-(3.10)$ is invariant under the change of $\bar{m}$ to $-\bar{m}$. Thus, we can assume $m>0$. Condition (3.10) together with (3.7) implies (3.6). The implication from (3.9) to (3.5) is trivial. This verifies the claim.

Let us reformulate the system (3.7)-(3.10) in matrix terms. We introduce the matrix

$$
B:=\left[\begin{array}{cccc}
\frac{1}{\beta_{0}} & & & -1 \\
& \ddots & & \vdots \\
& & \frac{1}{\beta_{t-1}} & -1 \\
-1 & \cdots & -1 & 1
\end{array}\right]
$$

of size $(t+1) \times(t+1)$ (the submatrix of $B$ generated by the first $t$ rows and $t$ columns is diagonal, with diagonal entries $\left.\frac{1}{\beta_{0}}, \ldots, \frac{1}{\beta_{t-1}}\right)$. The system (3.7)-(3.10) can thus be formulated as

$$
\left\{\begin{aligned}
\bar{m} & \in \mathbb{Z}^{t+1}, \\
\bar{m} & \neq o, \\
\|B \bar{m}\|_{\infty} & <1 .
\end{aligned}\right.
$$

We compute the determinant of $B$ by transforming the matrix into an upper triangular form: all -1 's in the last row can be turned to 0 by adding an appropriate linear combination of the first $t$ rows of $B$. Thus

$$
\operatorname{det} B=\frac{1-\sum_{i=0}^{t-1} \beta_{i}}{\prod_{j=0}^{t-1} \beta_{j}}=\frac{\sum_{i \in I} \beta_{i}}{\prod_{i \in J} \beta_{j}} .
$$

Hence $\operatorname{det} B \geq 0$ and since $I \neq \emptyset$, we even have $\operatorname{det} B>0$. We show $\operatorname{det} B \geq 1$ by contradiction. Assume that $\operatorname{det} B<1$. Then, by Theorem 2.2, (3.11) -(3.13) is solvable. The system (3.11)-(3.13) is a matrix form of (3.7)-(3.10). Above we showed that solvability of (3.7)-(3.10) implies solvability of (3.3)-(3.6). Thus, there exists $\bar{m}$ satisfying (3.3)-(3.6). With such $\bar{m}$ the lattice point $q$ given by (3.2) does not coincide with $p$ and lies in the interior of $T$. This yields a contradiction to $T \in \mathcal{T}^{d}$. Thus, $\operatorname{det} B \geq 1$ and the assertion follows from (3.14). 
Theorem 1.1 presents exponentially many inequalities for the barycentric coordinates of the interior lattice point: one for each $I$ with $\emptyset \varsubsetneqq I \varsubsetneqq\{0, \ldots, d\}$. It turns out that most of these inequalities are redundant. In fact, assume that for $i \in I$ and $j \in J$ one has $\beta_{i}>\beta_{j}$. Then we can modify $(I, J)$ by moving $i$ from $I$ to $J$ and moving $j$ from $J$ to $i$. By this change we lower the left hand side and raise the right hand side of (1.1) making the inequality tighter. We thus see that the inequalities for the partitions $(I, J)$ satisfying $\beta_{i} \leq \beta_{j}$ for every $i \in I$ and every $j \in J$ imply the inequalities for all the remaining partitions. Such strongest inequalities can be described as follows. After an appropriate reindexing, we can assume that the barycentric coordinates $\beta_{0}, \ldots, \beta_{d}$ are in an ordered sequence, say $\beta_{0} \geq \cdots \geq \beta_{d}>0$. Then, taking into account the redundancy, only the following $d$ inequalities remain:

$$
\sum_{i=j+1}^{d} \beta_{i} \geq \prod_{i=0}^{j} \beta_{i}
$$

where $j \in\{0, \ldots, d-1\}$.

\subsection{Lower bounds for $\beta_{i}$ 's}

Below we use (3.15) to derive lower bounds for the barycentric coordinates $\beta_{0}, \ldots, \beta_{d}$. First we observe that the inequalities $\beta_{0} \geq \cdots \geq \beta_{d}$ together with $\beta_{0}+\cdots+\beta_{d}=1$ yield $\beta_{0} \geq \frac{1}{d+1}$. Inequality (3.15) can be relaxed to

$$
(d+1) \beta_{j+1} \geq \prod_{i=0}^{j} \beta_{i}
$$

On the left hand side of (3.16) we could use a smaller factor instead of $d+1$, but with the factor $d+1$ the lower bounds for $\beta_{j}$ 's that we give below can be expressed by simpler formulas. By (3.16) each $\beta_{j}$ is bounded in terms of its 'predecessors' $\beta_{0}, \ldots, \beta_{j-1}$. Thus, consecutively applying (3.16) we can bound every $\beta_{j}$ in terms of $j$ and $\beta_{0}$. Taking into account $\beta_{0} \geq \frac{1}{d+1}$, we then see that each $\beta_{j}$ can be bounded from below in terms of $j$ and $d$. In this way we arrive at Part I of the following

Theorem 3.1. Let $d \in \mathbb{N}$. Then the following holds:

I. For every $T \in \mathcal{T}^{d}$ the (ordered) barycentric coordinates $\beta_{0} \geq \cdots \geq \beta_{d}>0$ of the unique interior lattice point of $T$ satisfy

$$
\beta_{i} \geq(d+1)^{-2^{i}}
$$

for every $i \in\{0, \ldots, d\}$.

II. There exists $T^{\prime} \in \mathcal{T}^{d}$ such that the barycentric coordinates $\beta_{0} \geq \cdots \geq \beta_{d}>0$ of the unique interior lattice point of $T^{\prime}$ satisfy

$$
\beta_{i} \leq \frac{1}{2^{2^{i-1}}-1}
$$

for every $i \in\{0, \ldots, d\}$. 
Part II of Theorem 3.1 is not used in the proof of the main result. By Part II we only wish to estimate the quality of the upper bounds in Part I.

Proof of Part I of Theorem 3.1. We use induction on $i$. For $i=0$, (3.17) is fulfilled. Assume the inequalities hold for all $i \in\{0, \ldots, j\}$ with some $j \in\{0, \ldots, d-1\}$. Using (3.16) and the inductive assumption we obtain

$$
\beta_{j+1} \geq(d+1)^{-1} \prod_{i=0}^{j}(d+1)^{-2^{i}}=(d+1)^{-\left(1+\sum_{i=0}^{j} 2^{i}\right)}=(d+1)^{-2^{j+1}} .
$$

Thus, (3.17) has been verified for $i=j+1$.

For showing Part II of Theorem 3.1] we use the following

Example 3.2. (A large simplex in $\mathcal{T}^{d}$.) Let $\mathbb{V}=\mathbb{R}^{d}$ and $\mathbb{L}=\mathbb{Z}^{d}$. Below we introduce the simplex $T^{\prime} \in \mathcal{T}^{d}$ given in [Hen83, $p$. 189]. The simplex $T^{\prime}$ is a slight modification of the simplex constructed by Zaks, Perles and Wills [ZPW82]. We first introduce a recursive sequence $\left(t_{n}\right)_{n \in \mathbb{N}}$ by

$$
t_{n}:= \begin{cases}2 & \text { for } n=1 \\ t_{n-1}^{2}-t_{n-1}+1 & \text { for } n \geq 2\end{cases}
$$

The sequence can also be defined by

$$
t_{n}= \begin{cases}2 & \text { for } n=1 \\ 1+\prod_{i=1}^{n-1} t_{i} & \text { for } n \geq 2\end{cases}
$$

The following relations for $t_{n}$ will be useful:

$$
\begin{aligned}
& 2^{2^{n-2}} \leq t_{n} \leq 2^{2^{n-1}} \\
& \frac{1}{t_{1}}+\ldots+\frac{1}{t_{n}}+\frac{1}{t_{n+1}-1}=1 .
\end{aligned}
$$

Relations (3.18), (3.19) and (3.20) can be found in [LZ91, p. 1026]. We define the simplex

$$
T^{\prime}:=\operatorname{conv}\left\{o, t_{1} e_{1}, \ldots, t_{d} e_{d}\right\} .
$$

Hensley [Hen83, p. 189] noticed that $e_{1}+\cdots+e_{d}$ is the unique interior lattice point of $T^{\prime}$. Thus, $T^{\prime} \in \mathcal{T}^{d}$. The bounds (3.19) show that $T^{\prime}$ is large for large $d$.

Proof of Part [I] of Theorem 3.1. Consider $T^{\prime}$ as in Example [3.2. From (3.20) we see that $\frac{1}{t_{1}}, \ldots, \frac{1}{t_{d}}$ and $\frac{1}{t_{d+1}-1}$ are the barycentric coordinates of the interior lattice point of $T^{\prime}$. The assertion follows from the lower bounds for $t_{n}$ given in (3.19).

Remark 3.3. For $T \in \mathcal{T}^{d}$ let $\beta_{d}(T)$ be the smallest barycentric coordinate of the interior lattice point of T. By Theorem 3.1. I. we show that

$$
c(d):=\inf _{T \in \mathcal{T}^{d}} \beta_{d}(T) \geq(d+1)^{-2^{d}} .
$$


From the arguments given in the remainder of the paper it will be seen that the finiteness of $\mathcal{T}^{d} / \operatorname{Aff}\left(\mathbb{Z}^{d}\right)$ is equivalent to the inequality $c(d)>0$. Pikhurko [Pik01, Section 7] indicates that $c(d)>0$ follows from the arguments of Lawrence [Law91, Lemma 5]. However, [Law91] does not yield any explicit positive lower bound for $c(d)$ for a general $d$.

Remark 3.4. (Quality of the lower bounds for $\beta_{i}$ 's.) The bound (3.17) is tight for $i=0$. This can be seen by considering the simplices $\operatorname{conv}\left\{o,(d+1) e_{1}, \ldots,(d+1) e_{d}\right\}$ and $\operatorname{conv}\left\{-\left(e_{1}+\cdots+e_{d}\right), e_{1}, \ldots, e_{d}\right\}$ (with respect to $\mathbb{L}=\mathbb{Z}^{d}$ ) whose unique interior lattice point is the center of mass. For large d's [LZ91, Lemma 2.2] provides the better bound $\beta_{d} \geq 14^{-2^{d+1}}$ than our bound $\beta_{d} \geq(d+1)^{-2^{d}}$ contained in (3.17). From this we see that the bound (3.17) seems to become less tight as $i$ moves from 0 to $d$. Still it should be mentioned that our lower bound for $\beta_{d}$ is better than the bound from [LZ91, Lemma 2.2] for $1 \leq d<14^{2}-1=195$.

The question on the determination of $c(d)$ given by (3.21) was raised by Hensley [Hen83, p. 189], who conjectured that the infimum in (3.21) is attained for the simplex $T^{\prime}$ from Example 3.2. This conjecture is true for simplices $T \in \mathcal{T}^{d}$ of the form $T=$ $\operatorname{conv}\left\{o, s_{1} e_{1}, \ldots, s_{d} e_{d}\right\}$ with $s_{1}, \ldots, s_{d} \in \mathbb{N}$, as follows from [Cur22] and [Erd50].

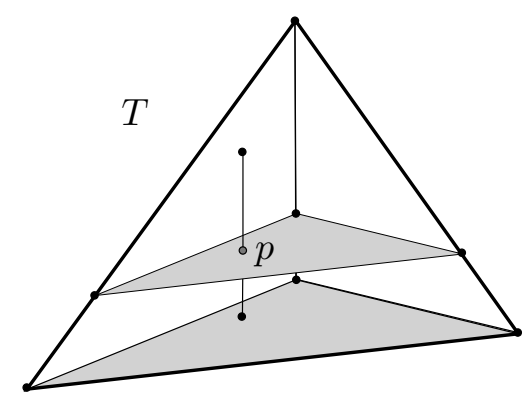

Figure 2: An interior point $p$ of a simplex $T$, a two-dimensional section of $T$ passing through $p$ and parallel to a facet of $T$ and a onedimensional section of $T$ passing through $p$ and parallel to an edge of $T$. Lemma 3.5.III shows how the volumes of such sections can be expressed in terms of the volumes of the faces of $T$ and the barycentric coordinates of $p$.

\subsection{Face volumes versus $\beta_{i}$ 's}

In the following lemma we discuss affine sections of $T$ passing through a fixed interior point of $T$ and parallel to faces of $T$, for an illustration see Fig. 2. We shall use the affine-linear functions defined by (2.3) .

Lemma 3.5. Let $T$ be a d-dimensional simplex in $\mathbb{V}$ with vertices $p_{0}, \ldots, p_{d}$. Let $p$ be a point in int $T$ with barycentric coordinates $\beta_{0}, \ldots, \beta_{d}$. Let $I, J$ be disjoint sets with 
$I \cup J=\{0, \ldots, d\}$ and $J \neq \emptyset$ and let

$$
\begin{aligned}
& T_{I}:=\left\{x \in T: l_{i}(x)=\beta_{i} \quad \forall i \in I\right\}, \\
& \beta_{J}:=\sum_{j \in J} \beta_{j} .
\end{aligned}
$$

Then the following holds:

I. The set $T_{I}$ is a simplex of dimension $|J|-1$, and the points $\sum_{i \in I} \beta_{i} p_{i}+\beta_{J} p_{j}$ with $j \in J$ are the vertices of $T_{I}$.

II. If $x \in T_{I}$, then the values $\frac{l_{j}(x)}{\beta_{J}}$ with $j \in J$ are the barycentric coordinates of $x$ with respect to $T_{I}$.

III. One has $\operatorname{vol}\left(T_{I}\right)=\beta_{J}^{|J|-1} \operatorname{vol}\left(F_{I}\right)$, where $F_{I}$ is defined by (2.4).

Proof. Let $x \in T_{I}$. The values $\frac{l_{j}(x)}{\beta_{J}}$ with $j \in J$ satisfy

$$
\sum_{j \in J} \frac{l_{j}(x)}{\beta_{J}}=\frac{1}{\beta_{J}}\left(1-\sum_{i \in I} \beta_{i}\right)=1 .
$$

Furthermore

$$
\begin{aligned}
x=\sum_{i=0}^{d} l_{i}(x) p_{i} & =\sum_{i \in I} \beta_{i} p_{i}+\sum_{j \in J} l_{j}(x) p_{j} \\
& =\sum_{j \in J} \frac{l_{j}(x)}{\beta_{J}}\left(\sum_{i \in I} \beta_{i} p_{i}+\beta_{J} p_{j}\right) .
\end{aligned}
$$

Thus $x$ is a convex combination of the points $\sum_{i \in I} \beta_{i} p_{i}+\beta_{J} p_{j}$ with $j \in J$. These $|J|$ points are affinely independent since they are obtained by applying the nonsingular affine transformation $y \mapsto \sum_{i \in I} \beta_{i} p_{i}+\beta_{J} y(y \in \mathbb{V})$ to the affinely independent points $p_{j}$ with $j \in J$. This yields Parts I and II. Part III follows in view of the equality $F_{I}=\operatorname{conv}\left\{p_{j}: j \in J\right\}$.

The following assertion appears in [Pik01, Lemma 5].

Lemma 3.6. Let $T$ be a d-dimensional simplex in $\mathbb{V}$ such that the interior of $T$ contains precisely one point of $\mathbb{L}$. Let $\beta_{0}, \ldots, \beta_{d}>0$ be the barycentric coordinates of the unique interior lattice point of $T$ and let $N$ be a subset of $\{0, \ldots, d\}$ of cardinality $d$. Then

$$
\operatorname{vol}_{\mathbb{L}}(T) \leq \frac{1}{d ! \prod_{n \in N} \beta_{n}}
$$

Proof. Let $p$ be the lattice point in the interior of $T$. We have $p=\sum_{i=0}^{d} \beta_{i} p_{i}$, where $p_{i}$ 's are appropriately indexed vertices of $T$. Without loss of generality assume $N=$ $\{1, \ldots, d\}$. Consider affine-linear functions $l_{0}, \ldots, l_{d}$ defined by (2.3). We have $l_{i}(p)=\beta_{i}$ 


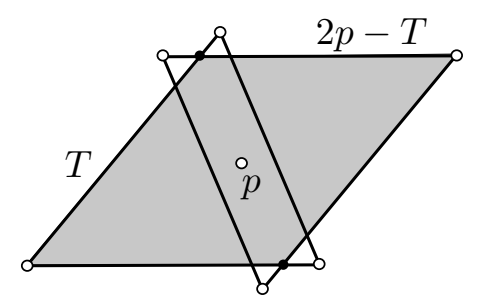

Figure 3: Illustration to the proof of Lemma 3.6. The center of the symmetry $p$ of the parallelotope $P$ (shaded) is the unique interior lattice point of $P$

for $i \in\{0, \ldots, d\}$. The simplex $\tilde{T}:=2 p-T$ is a reflection of $T$ in $p ; \tilde{T}$ can be described as

$$
\tilde{T}:=\left\{x \in \mathbb{R}^{d}: \tilde{l}_{0}(x) \geq 0, \ldots, \tilde{l}_{d}(x) \geq 0\right\}
$$

where $\tilde{l}_{i}$ 's are defined by $\tilde{l}_{i}(x):=l_{i}(2 p-x)$ for $x \in \mathbb{R}^{d}$ and $i \in\{0, \ldots, d\}$. By affinelinearity of $l_{0}$, for every $x \in \mathbb{V}$ we have

$$
0<\beta_{0}=l_{0}(p)=\frac{1}{2}\left(l_{0}(x)+l_{0}(2 p-x)\right)=\frac{1}{2}\left(l_{0}(x)+\tilde{l}_{0}(x)\right) .
$$

Consequently, for every $x \in \mathbb{V}, l_{d}(x) \geq 0$ or $\tilde{l}_{d}(x) \geq 0$. The set

$$
P:=\left\{x \in \mathbb{R}^{d}: l_{i}(x) \geq 0 \text { and } \tilde{l}_{i}(x) \geq 0 \text { for all } i=1, \ldots, d\right\}
$$

is a parallelotope with center at $p$. Let us show $P \subseteq T \cup \tilde{T}$ (see also Fig. 3):

$$
\begin{aligned}
P:= & \left\{x \in \mathbb{R}^{d}: l_{1}(x) \geq 0, \ldots, l_{d}(x) \geq 0, \tilde{l}_{1}(x) \geq 0, \ldots, \tilde{l}_{d}(x) \geq 0\right\} \\
= & \left\{x \in \mathbb{R}^{d}: l_{1}(x) \geq 0, \ldots, l_{d}(x) \geq 0, \tilde{l}_{1}(x) \geq 0, \ldots, \tilde{l}_{d}(x) \geq 0\right. \\
& \text { and } \left.\left(l_{0}(x) \geq 0 \text { or } \tilde{l}_{0}(x) \geq 0\right)\right\} \\
= & \left\{x \in \mathbb{R}^{d}: l_{0}(x) \geq 0, \ldots, l_{d}(x) \geq 0, \tilde{l}_{1}(x) \geq 0, \ldots, \tilde{l}_{d}(x) \geq 0\right\} \\
& \cup\left\{x \in \mathbb{R}^{d}: l_{1}(x) \geq 0, \ldots, l_{d}(x) \geq 0, \tilde{l}_{0}(x) \geq 0, \ldots, \tilde{l}_{d}(x) \geq 0\right\} \\
& \subseteq T \cup \tilde{T} .
\end{aligned}
$$

Since $p$ is the only interior lattice point of both $T$ and $\tilde{T}$, we deduce that $p$ is the only interior lattice point of $P$. Due to 'affine invariance' of the ratio $\operatorname{vol}(T) / \operatorname{vol}(P)$ for computing $\operatorname{vol}(T) / \operatorname{vol}(P)$ we can assume that $\mathbb{V}=\mathbb{R}^{d}$ and $p_{0}=o, p_{1}=e_{1}, \ldots, p_{d}=e_{d}$. With this assumption we get $p=\left(\beta_{1}, \ldots, \beta_{d}\right)^{\top}$ and $P$ is precisely the set of vectors $x=\left(x_{1}, \ldots, x_{d}\right)^{\top}$ for which $0 \leq x_{i} \leq 2 \beta_{i}$. Then $\operatorname{vol}(P)=2^{d} \prod_{i=1}^{d} \beta_{i}, \operatorname{vol}(T)=\frac{1}{d !}$ and we 
obtain $\operatorname{vol}(T) / \operatorname{vol}(P)=\frac{1}{d ! 2^{d} \prod_{i=1}^{d} \beta_{i}}$. By Minkowski's fundamental theorem, $\operatorname{vol}_{\mathbb{L}}(P) \leq$ $2^{d}$. Hence

$$
\begin{aligned}
\operatorname{vol}_{\mathbb{L}}(T) & =\frac{\operatorname{vol}(T)}{\operatorname{det} \mathbb{L}}=\frac{\operatorname{vol}(T)}{\operatorname{vol}(P)} \cdot \operatorname{vol}_{\mathbb{L}}(P) \\
& \leq \frac{\operatorname{vol}(T)}{\operatorname{vol}(P)} 2^{d}=\frac{1}{d ! 2^{d} \prod_{i=1}^{d} \beta_{i}} 2^{d}=\frac{1}{d ! \prod_{i=1}^{d} \beta_{i}}
\end{aligned}
$$

The following theorem follows directly from Lemma 3.6.

Theorem 3.7. Let $T \in \mathcal{T}^{d}$ and $\beta_{0}, \ldots, \beta_{d}$ be the barycentric coordinates of the interior lattice point of $T$. Let $I$ and $N$ be disjoint sets such that $I \cup N$ is a d-element subset of $\{0, \ldots, d\}$. Then

$$
\operatorname{vol}_{\mathbb{L}}\left(F_{I}\right) \leq \frac{1}{|N| ! \prod_{n \in N} \beta_{n}}
$$

Proof. Let $J:=\{0, \ldots, d\} \backslash I$. By Lemma 3.5.III we have $\operatorname{vol}_{\mathbb{L}}\left(F_{I}\right)=\beta_{J}^{1-|J|} \operatorname{vol}_{\mathbb{L}}\left(T_{I}\right)$ with $T_{I}$ and $\beta_{J}$ defined by (3.22) and (3.23), respectively. By Lemma 3.5.II, the interior lattice point of $T$ has barycentric coordinates $\frac{\beta_{j}}{\beta_{J}}$ with $j \in J$ with respect to the simplex $T_{I}$. Thus, applying Lemma 3.6 (for dimension $|J|-1$ ) to the $(|J|-1$ )-dimensional simplex $T_{I}$ we get

$$
\operatorname{vol}_{\mathbb{L}}\left(F_{I}\right)=\beta_{J}^{1-|J|} \operatorname{vol}_{\mathbb{L}}\left(T_{I}\right) \leq \beta_{J}^{1-|J|} \frac{1}{|N| ! \prod_{n \in N} \frac{\beta_{n}}{\beta_{J}}}=\frac{1}{|N| ! \prod_{n \in N} \beta_{n}} .
$$

\subsection{The conclusion}

Proof of Theorem 1.2. First we show Part I. Let $\beta_{0} \geq \cdots \geq \beta_{d}>0$ be the barycentric coordinates of the unique interior lattice point of $T$. For $i \in\{1, \ldots, d\}$ we define $G_{i}$ by $G_{i}:=F_{I}$, where $I:=\{0, \ldots, d\} \backslash\{0, \ldots, i\}$. By Theorems 3.1 and 3.7 we obtain

$$
\operatorname{vol}_{\mathbb{L}}\left(G_{i}\right)=\operatorname{vol}_{\mathbb{L}}\left(F_{I}\right) \leq \frac{1}{i ! \prod_{n=0}^{i-1} \beta_{n}} \leq \frac{1}{i !} \prod_{n=0}^{i-1}(d+1)^{2^{n}}=\frac{1}{i !}(d+1)^{2^{i}-1} .
$$

This yields (1.2). Inequality (1.3) follows directly from (1.2) and Blichfeldt's theorem.

For showing Part II we consider $\mathbb{L}=\mathbb{Z}^{d}$ and the simplex $T^{\prime}$ from Example 3.2. For $i \in\{1, \ldots, d\}$ let $G_{i}:=\operatorname{conv}\left\{o, t_{1} e_{1}, \ldots, t_{i} e_{i}\right\}$. Applying (3.18) and (3.19) we obtain

$$
\operatorname{vol}_{\mathbb{L}}\left(G_{i}\right)=\frac{1}{i !} \prod_{j=1}^{i} t_{j}=\frac{1}{i !}\left(t_{i+1}-1\right) \geq \frac{1}{i !}\left(2^{2^{i-1}}-1\right) .
$$

This shows (1.4). Since $G_{i} \supseteq\left\{j e_{i}: j=0, \ldots, t_{i}\right\}$, we have $\left|G_{i} \cap \mathbb{L}\right| \geq t_{i}$. The bound (1.5) follows after applying (3.19). 
Acknowledgements. I would like to thank A. M. Kasprzyk, Ch. Wagner and the anonymous referees for pointers to the literature and for comments that helped to improve the presentation.

\section{References}

[AWW11] G. Averkov, Ch. Wagner, and R. Weismantel, Maximal lattice-free polyhedra: finiteness and an explicit description in dimension three, Math. Oper. Res. 36 (2011), no. 4, 721-742.

[Bar02] A. Barvinok, A Course in Convexity, Graduate Studies in Mathematics, vol. 54, American Mathematical Society, Providence, RI, 2002.

[BB92] A. A. Borisov and L. A. Borisov, Singular toric Fano three-folds, Mat. Sb. 183 (1992), no. 2, 134-141.

[Bor00] A. Borisov, Convex lattice polytopes and cones with few lattice points inside, from a birational geometry viewpoint, Preprint ArXiv:math/0001109, 2000.

[Cur22] D. R. Curtiss, On Kellogg's Diophantine Problem, Amer. Math. Monthly 29 (1922), no. 10, 380-387.

[DPW10] A. Del Pia and R. Weismantel, On convergence in mixed integer programming, Preprint, to appear in Math. Programming, 2010.

[Ehr55] E. Ehrhart, Une généralisation du théorème de Minkowski, C. R. Acad. Sci. Paris 240 (1955), 483-485.

[Erd50] P. Erdős, On a Diophantine equation, Mat. Lapok 1 (1950), 192-210.

[GL87] P. M. Gruber and C. G. Lekkerkerker, Geometry of Numbers, second ed., North-Holland Mathematical Library, vol. 37, North-Holland Publishing Co., Amsterdam, 1987.

[Gru07] P. M. Gruber, Convex and Discrete Geometry, Grundlehren der Mathematischen Wissenschaften [Fundamental Principles of Mathematical Sciences], vol. 336, Springer, Berlin, 2007.

[Hen83] D. Hensley, Lattice vertex polytopes with interior lattice points, Pacific J. Math. 105 (1983), no. 1, 183-191.

[HNP09] Ch. Haase, B. Nill, and S. Payne, Cayley decompositions of lattice polytopes and upper bounds for $h^{*}$-polynomials, J. Reine Angew. Math. 637 (2009), 207-216.

[HS09] Ch. Haase and J. Schicho, Lattice polygons and the number $2 i+7$, Amer. Math. Monthly 116 (2009), no. 2, 151-165.

[Kas09] A. M. Kasprzyk, Bounds on fake weighted projective space, Kodai Math. J. 32 (2009), no. 2, 197-208. 
[Kas10] Canonical toric Fano threefolds, Canad. J. Math. 62 (2010), no. 6, 1293-1309.

[Law91] J. Lawrence, Finite unions of closed subgroups of the $n$-dimensional torus, Applied geometry and discrete mathematics, DIMACS Ser. Discrete Math. Theoret. Comput. Sci., vol. 4, Amer. Math. Soc., Providence, RI, 1991, pp. 433441.

[LZ91] J. C. Lagarias and G. M. Ziegler, Bounds for lattice polytopes containing a fixed number of interior points in a sublattice, Canad. J. Math. 43 (1991), no. 5, 1022-1035.

[NZ11] B. Nill and G. M. Ziegler, Projecting lattice polytopes without interior lattice points, Math. Oper. Res. 36 (2011), no. 3, 468-473.

[Pik01] O. Pikhurko, Lattice points in lattice polytopes, Mathematika 48 (2001), no. 12, 15-24 (2003). MR 2004f:52009

[Rab89] S. Rabinowitz, A census of convex lattice polygons with at most one interior lattice point, Ars Combin. 28 (1989), 83-96.

[Sch93] R. Schneider, Convex Bodies: The Brunn-Minkowski Theory, Encyclopedia of Mathematics and its Applications, vol. 44, Cambridge University Press, Cambridge, 1993.

[Sco76] P. R. Scott, On convex lattice polygons, Bull. Austral. Math. Soc. 15 (1976), no. 3, 395-399.

[ZPW82] J. Zaks, M. A. Perles, and J. M. Wills, On lattice polytopes having interior lattice points, Elem. Math. 37 (1982), no. 2, 44-46. 